\title{
Clinical Approach to Skin Eruption and Measles: A Mini Review
}

Ayse Batirel ${ }^{1}$ and Mehmet Doganay ${ }^{2 *}$

${ }^{1}$ Department of Infectious Diseases and Clinical Microbiology, Kartal Dr. Lutfi Kirdar Education and Research Hospital, Istanbul, Turkey

${ }^{2}$ Department of Infectious Diseases, Faculty of Medicine, Erciyes University, Kayseri, Turkey

\begin{abstract}
Different etiologic agents or diseases may cause similar types of skin eruption or a specific disease may present with different types of skin eruptions. Because of a wide spectrum of causes of generalized skin eruption, differential diagnosis poses a challenge to physicians. Viral infections more often cause symmetrical rashes widely distributed all over the body which are self-limited and usually last up to one week. Most common causes of maculopapular rashes are viral exanthems and drug reactions in children and in adults, respectively. Measles seems to be controlled in industrial countries with the strict vaccination; it is still endemic/epidemic in developing countries. Measles is reemerged in some countries where measles was controlled before for the reason of uncontrolled migration, fighting, poverty, infrastructure in health service. In this paper, we aimed to review clinical approach to skin eruption and measles briefly.
\end{abstract}

Keywords: Skin eruption; Measles; Rash; Fever; Exanthem

\section{Introduction}

Various character skin eruptions can be seen due to drug reaction, infectious diseases (viral, bacterial, fungal and parasitic diseases), autoimmune and rheumatologic diseases, malignant diseases, insect bites etc. Measles is a viral infection and commonly seen in childhood period. On the other hand, the disease may be seen rarely in adult age group and leads to diagnostic difficulties. The main manifestation of measles is fever and skin eruption and may mimic many diseases. The patients having skin eruptions are firstly apply to family physicians or first step health care centers. Measles seems to be controlled in industrial countries with the strict vaccination; it is still endemic/epidemic in developing countries. Measles is also re-emerged in some countries where measles was controlled before for the reason of uncontrolled migration, fighting, poverty, infrastructure in health service. Currently many physicians are not familiar to clinical presentation of measles. In this paper, we aimed to remind clinical approach to skin eruptions and review measles briefly.

\section{Clinical approach to skin eruption}

Skin eruption is any eruption located on the skin that occurs as a disease symptom. Various infectious (bacterial, viral, fungal, parasitic infections) (50\%) and non-infectious (dermatologic conditions such as atopic / contact dermatitis, erythema multiforme, allergic reactions, collagen vascular diseases, liver or kidney disease, some cancer types, drugs) (40\%) causes lead to different types of skin eruptions. In 10$30 \%$ of cases, the etiology of skin eruption cannot be diagnosed despite a detailed investigation [1,2]. Different etiologic agents or diseases may cause similar types of skin eruption or a specific cause/disease may present with different types of skin eruptions. Because of such a wide spectrum of causes of generalized skin eruption, differential diagnosis poses a challenge [2]. Nevertheless, prompt correct clinical diagnosis is necessary to decide on immediate treatment especially if the clinical status is severe or life-threatening (e.g. febrile patients with toxic appearance, meningococcemia, infective endocarditis, toxic shock syndrome, toxic epidermal necrolysis) [3], moreover, patients with skin eruptions due to contagious infections need to be isolated to prevent transmission to others.

For an appropriate clinical approach to patients with skin eruption, a detailed medical and epidemiological history including travel history a thorough physical examination, basic hematologic, biochemical and microbiologic tests are required. In the differential diagnosis of skin eruption, important clinical features are; the type / morphology (maculopapular, petechial, purpuric, vesicular, vesiculobullous, diffuse erythematous, or nodular), characteristics (size, color, shape, pain/ tenderness, pruritus, scaling, erythema, blanching on pressure etc.), location and distribution on the body (involved and spared areas of the body, i.e. mucous membranes, palms and soles, scalp, face etc.), initiation time, duration, change in morphology or progression, factors stimulating or diminishing it, presence or absence of fever or contact with a febrile patient with rash, prodromal symptoms or concomitant other systemic symptoms and findings, desquamation, seasonal relationship; which may all give a clue about the cause. In a patient with skin eruption; his/her occupation, any drug use in previous 30 days, drug allergy, family history of allergic diseases, recent travel history, contact with animals, insects, vaccination status, previous exanthematous illnesses, sun exposure, chemical or environmental exposure should be questionized to obtain a detailed medical history. Underlying diseases such as immunosuppresion, transplantation, corticosteroid or chemotherapeutic use, asplenia, cardiac valve disease should also be noted carefully.

On physical examination; general appearance and vital signs of the patient, presence or absence of lymphadenopathy, arthritis, hepatosplenomegaly, mucosal, genital and/or conjunctival lesions, meningeal irritation signs, some pathognomonic signs or distinctive/ key clinical characteristics (e.g. Koplik spots in measles) may help in differential diagnosis of the disease with skin eruption. If the clinical diagnosis is unclear and the patient does not respond to initial symptomatic treatment and/or the skin eruption does not resolve,

*Corresponding author: Mehmet Doganay M.D, Professor in Infectious Diseases, Faculty of Medicine, Erciyes University, 38039 Kayseri, Turkey, E-mail: mdoganay@erciyes.edu.tr

Received July 12, 2013; Accepted July 31, 2013; Published August 05, 2013

Citation: Batirel A, Doganay M (2013) Clinical Approach to Skin Eruption and Measles: A Mini Review. J Gen Pract 1: 118. doi: 10.4172/2329-9126.1000118

Copyright: ( 2013 Batirel A, et al. This is an open-access article distributed under the terms of the Creative Commons Attribution License, which permits unrestricted use, distribution, and reproduction in any medium, provided the original author and source are credited. 
further diagnostic laboratory tests, consultation by dermatologists and/ or skin biopsy may be needed $[4,5]$.

\section{Definitions and examples of different types of skin eruption}

Macule: A (nonpalpable) change in skin color without elevation or depression of the skin surface (eg: viral exanthems, atopic dermatitis, drug eruptions, rosacea, vitiligo, Kawasaki's disease).

Papule: A solid, raised, palpable skin lesion less than $0.5 \mathrm{~cm}$ in diameter (eg: viral exanthems, Scarlet fever, atopic dermatitis, drug eruption, insect bites).

Nodule: A lesion similar to but larger than a papule invading skin and subcutaneous tissue (eg: nodulocystic acne).

Plaque: A broad, slightly raised skin lesion with a size of involvement larger than its height (eg: psoriasis, lichen planus)

Pustule: A vesicle that involves purulent fluid (eg: folliculitis, impetigo, hand-foot-and mouth disease, Behçet's disease, candidiasis).

Vesicle: A lesion smaller than $0.5 \mathrm{~cm}$ in diameter and involving serous fluid (eg: Varicella, Herpes virus infections).

Bullae: Vesicles larger than $0.5 \mathrm{~cm}$ in diameter (eg: toxic epidermal necrolysis, Staphylococcal scalded skin syndrome, bullous pemphigoid, bullous impetigo).

Petechiae: Non-blanching lesions smaller than $3 \mathrm{~mm}$ in diameter which involve extravasated erythrocytes or hemoglobin (eg: meningococcemia, Scarlet fever, sepsis, viral hemorrhagic fevers, cytomegalovirus and Parvovirus B19 infections, vasculitis, hemolytic uremic syndrome).

Purpura: Petechiae larger than $3 \mathrm{~mm}$ in diameter (eg: meningococcemia, thrombocytopenia, idiopathic thrombocytopenic purpura, leptospirosis, Rickettsia, Neisseria gonorrhoea).

Polymorphic skin lesion: involves more than one morphologic type of skin eruption (eg: Varicella).

\section{An overview of measles}

Measles seems to be controlled in industrial countries with the strict vaccination; it is still endemic/ epidemic in developing countries. Measles is re-emerged in some countries where measles was controlled before for the reason of uncontrolled migration, fighting, poverty, infrastructure in health service.

After the introduction of measles vaccine and due to high vaccine coverage, there has been a decline in measles cases in the western world until 2009. Recently, re-emergence of measles has been observed in Europe due to sub-optimal immunization and measles cases increased four fold between 2010 and 2011 [6,7]. Also, the number of cases has increased recently in other developed countries such as US and outbreaks of measles still occur [8,9]. It is still one of the major causes of morbidity and mortality in childhood mostly because of secondary infections resulting from a measles-induced transient suppression of immune response [10]. Despite the availability of an effective and safe vaccine, in 2011, 158000 measles deaths (approximately 430 deaths every day or 18 deaths every hour) were reported globally by WHO, with more than $95 \%$ of deaths occurring in developing countries. Vaccination resulted in a $71 \%$ decrease in deaths due to measles between 2000 and 2011. More than 20 million people are infected by measles virus each year. In measles-eliminated countries, imported cases of measles constitute a major source of infection [11].

\section{Characteristic findings of measles}

Viral infections more often cause symmetrical rashes widely distributed all over the body which are self-limited and usually last up to one week. Most common causes of maculopapular rashes are viral exanthems and drug reactions in children and in adults, respectively $[1,2]$. Although regarded as a highly contagious disease of childhood mostly, Measles (Rubeola) presents a more severe course in adults. In developing countries, mainly children are affected, but in developed countries, the disease occurs in adolescents and adults.

It has an incubation period of 10 to 14 days followed by a prodromal phase. High fever (lasting 3-4 days), anorexia, malaise, sorethroat, conjunctivitis, coryza, cough and Koplik's Spots (KS) are major symptoms and signs during prodromal phase. KS (first described by Koplik in 1896) are the enanthems resembling "grains of sand" that appear on buccal mucosa opposite to the second molar teeth before rash occurs, and they constitute the pathognomonic finding of measles. As the erythematous maculopapular rash of measles (which usually starts on the face and hairline on the neck) appears, Koplik's spots disappear. Within about three days, the rash spreads downwards (from cranial to caudal direction) to involve the trunk (Figure 1), and then the extremities, rarely the palms and soles and tends to be confluent on the neck and face. It usually lasts approximately 5-7 days. Desquamation may occur during the healing period [12]. Dermatological manifestations of measles may be heterogeneous and may differ from one case to another [13].

Patients are contagious four days prior to and four days after the onset of rash. The fever usually resolves after several days of rash. If it persists for more than 3-4 days after the onset of rash, it may suggest presence of a complication. In children less than 5 and adults older than 20 years, the incidence of complications due to measles has been reported to be higher. Among complications, otitis media is the most common one, while bacterial respiratory superinfections are the most common causes of death in severe measles. Subacute sclerosing panencephalitis is a less common but serious complication. Bronchospasm, sinusitis, hepatitis, diarrhea, keratitis, blindness in case of vitamin A deficiency and post-infectious encephalitis are other reported complications of measles [12]. Infection with measles virus during pregnancy may cause severe complications such as abortus or preterm delivery [14].

In previously vaccinated patients or infants with maternal antibody

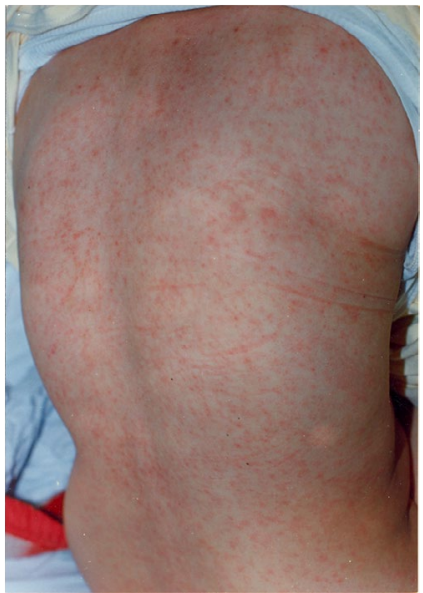

Figure 1: Typical skin eruption in an adult patient with measles. The picture is obtained from Prof. Mehmet Doganay. 
to measles who have partial immunity to measles, a mild form of disease called "Modified Measles" may be observed. Typical symptoms of measles may be absent in this mild, modified form which may be subclinical [15].

"Atypical Measles", which has a more severe and prolonged clinical course, has been described in patients who previously received killed measles vaccine and were exposed to measles virus, but have incomplete immunity to measles [16]. High fever lasting 2-4 days, headache, cough, a maculopapular rash starting peripherally from hands and feet, spreading to the body and face (which differs from "classic" measles in that aspect), petechial and/or purpuric, urticarial, rarely vesicular or hemorrhagic rash, pneumonia with interstitial pulmonary infiltrates, and hepatitis may be observed in this form of measles.

In immunocompromised [e.g. in case of treatment for malignant disease, transplantation, congenital immunodeficiency or acquired immunodeficiency syndrome (AIDS)] and poorly nourished patients (e.g.: vitamin A insufficiency), measles may be more severe (with pneumonia and chronic encephalitis) with a mortality of $40-70 \%$ $[12,17]$ and difficult to diagnose.

\section{Diagnosis of measles}

Despite availability of highly sensitive laboratory tests which may take a few days to obtain the results, diagnosis and management of measles is particularly based on clinical presentation and suspicion. Moreover, control measures should be taken promptly usually in absence of laboratory confirmation, as the disease is highly contagious by transmission of the virus by droplets via coughing, sneezing, direct contact with infected secretions and/or close personal contact.

Clinical diagnosis may be easy in presence of pathognomonic KS and in areas with high measles incidence. Using KS as a diagnostic tool increases the Positive Predictive Value (PPV) of clinical diagnosis from $50 \%$ to $80 \%$. KS were found to be highly predictive of confirmed measles [18].

Main laboratory findings include leukopenia, thrombocytopenia and elevation of liver transaminases. A laboratory diagnosis may be necessary in cases of atypical measles or in immunocompromised individuals. Laboratory diagnosis of measles includes demonstration of specific IgM antibody which is recommended by WHO as the standard test for surveillance of measles or a fourfold or greater increase in measles IgG antibody titer in acute and convalescent sera by ELISA [19]. Neutralization, complement fixation, immunofluorescent microscopy, virus isolation, identification of measles antigen or RNA in infected tissues by RT-PCR amplification is other diagnostic methods. Measles virus can be detected from nasopharyngeal swab, urine or blood after the onset of rash by PCR [20].

Scarlet fever, other viral exanthems like rubella, roseola, adenoviral, enteroviral and rickettsial infections, infectious mononucleosis, toxic shock syndrome, drug eruptions should be considered in the differential diagnosis of measles. Primary infections due to Human Herpes Virus (HHV)-6 (20\%) or HHV-7 (8\%) can be misdiagnosed as measles or rubella based on clinical presentation [21]. Multiplex real-time PCR may be a useful screening tool for detection and differentiation of rashassociated viruses such as Measles virus, Rubella virus, Parvovirus B19, HHV-6 and HHV- in clinical specimens [22]. False-positivity of IgM tests by indirect enzyme immunoassay (EIA) kits can occur because of rubella, Parvovirus B19, and HHV-6 infection [23]. A positive result of measles IgM should be interpreted in association with clinical and epidemiological data [24]. Also, oral fluid tests may be used for the differential diagnosis [25].

\section{Treatment of measles}

No specific anti-viral therapy is available. Therefore, supportive care with adequate food and fluid intake, and antipyretics is imperative/ crucial to avoid complications. Secondary bacterial infections such as otitis media, pneumonia should be treated with antibiotics [26]. Two doses of vitamin A supplements should be given to children to prevent development of severe complications such as visual impairment, blindness and death [27]. Most patients recover within 2-3 weeks if the disease is uncomplicated.

\section{Prevention}

Immunization with two doses of measles vaccine in combination with rubella and mumps vaccines (MMR) is an effective and safe method of prevention [28]. About $15 \%$ of children vaccinated with only one dose fail to develop complete immunity against measles. Therefore, two doses of measles vaccine are recommended to obtain complete immunity and prevent outbreaks. At least $95 \%$ of the population should be vaccinated with two doses of MMR vaccine for elimination of measles [6].

\section{References}

1. Tabak F, Murtezaoglu A, Tabak O, Ozaras R, Mete B, et al. (2012) Clinical features and etiology of adult patients with Fever and rash. Ann Dermatol 24: 420-425.

2. Drago F, Rampini E, Rebora A (2002) Atypical exanthems: morphology and laboratory investigations may lead to an aetiological diagnosis in about $70 \%$ of cases. Br J Dermatol 147: 255-260.

3. Drage LA (1999) Life-threatening rashes: dermatologic signs of four infectious diseases. Mayo Clin Proc 74: 68-72.

4. Ely JW, Seabury Stone M (2010) The generalized rash: part I. Differential diagnosis. Am Fam Physician 81: 726-734.

5. Ely JW, Seabury Stone M (2010) The generalized rash: part II. Diagnostic approach. Am Fam Physician 81: 735-739.

6. Carrillo-Santisteve P, Lopalco PL (2012) Measles still spreads in Europe: who is responsible for the failure to vaccinate? Clin Microbiol Infect 18 Suppl 5: 50-56.

7. Leuridan E, Sabbe M, Van Damme P (2012) Measles outbreak in Europe: susceptibility of infants too young to be immunized. Vaccine 30: 5905-5913.

8. Sugerman DE, Barskey AE, Delea MG, Ortega-Sanchez IR, Bi D, et al. (2010) Measles outbreak in a highly vaccinated population, San Diego, 2008: role of the intentionally undervaccinated. Pediatrics 125: 747-755.

9. Sabella C (2010) Measles: not just a childhood rash. Cleve Clin J Med 77 207-213.

10. Griffin DE, Lin WH, Pan CH (2012) Measles virus, immune control, and persistence. FEMS Microbiol Rev 36: 649-662.

11. http://www.who.int/mediacentre/factsheets/fs286/en/

12. Perry RT, Halsey NA (2004) The clinical significance of measles: a review. J Infect Dis 189 Suppl 1: S4-S16.

13. Battegay R, Itin C, Itin P (2012) Dermatological signs and symptoms of measles: a prospective case series and comparison with the literature. Dermatology 224: 1-4.

14. White SJ, Boldt KL, Holditch SJ, Poland GA, Jacobson RM (2012) Measles, mumps, and rubella. Clin Obstet Gynecol 55: 550-559.

15. Krugman S, Giles JP, Friedman H, Stone S (1965) Studies on Immunity to Measles. J Pediatr 66: 471-88.

16. Polack FP, Hoffman SJ, Crujeiras G, Griffin DE (2003) A role for nonprotective complement-fixing antibodies with low avidity for measles virus in atypical measles. Nat Med 9: 1209-1213. 
Citation: Batirel A, Doganay M (2013) Clinical Approach to Skin Eruption and Measles: A Mini Review. J Gen Pract 1: 118. doi: 10.4172/23299126.1000118

17. Kaplan LJ, Daum RS, Smaron M, McCarthy CA (1992) Severe measles in immunocompromised patients. JAMA 267: 1237-1241.

18. Zenner D, Nacul L (2012) Predictive power of Koplik's spots for the diagnosis of measles. J Infect Dev Ctries 6: 271-275.

19. Bellini WJ, Helfand RF (2003) The challenges and strategies for laboratory diagnosis of measles in an international setting. J Infect Dis 187 Suppl 1: S283-S290.

20. Matsuzono Y, Narita M, Ishiguro N, Togashi T (1994) Detection of measles virus from clinical samples using the polymerase chain reaction. Arch Pediatr Adolesc Med 148: 289-293.

21. Black JB, Durigon E, Kite-Powell K, de Souza L, Curli SP, et al. (1996) Seroconversion to human herpesvirus 6 and human herpesvirus 7 among Brazilian children with clinical diagnoses of measles or rubella. Clin Infect Dis 23: $1156-1158$

22. Kaida A, Kubo H, Sekiguchi J, Ohyama M, Goto K, et al. (2012) Detection of five rash-associated viruses using multiplex real-time PCR during 2006-2011. Jpn J Infect Dis 65: 430-432.

23. Ciccone FH, Carvalhanas TR, Afonso AM, Flannery B, Waldman EA (2010)
Investigation of measles IgM-seropositive cases of febrile rash illnesses in the absence of documented measles virus transmission, State of São Paulo, Brazil, 2000-2004. Rev Soc Bras Med Trop 43: 234-239.

24. Dietz V, Rota J, Izurieta H, Carrasco P, Bellini W (2004) The laboratory confirmation of suspected measles cases in settings of low measles transmission: conclusions from the experience in the Americas. Bull World Health Organ 82: 852-827.

25. Ramsay M, Reacher M, O'Flynn C, Buttery R, Hadden F, et al. (2002) Causes of morbilliform rash in a highly immunised English population. Arch Dis Child 87: 202-206

26. Kabra SK, Lodha R, Hilton DJ (2008) Antibiotics for preventing complications in children with measles. Cochrane Database Syst Rev 16: CD001477.

27. Melenotte C, Brouqui P, Botelho-Nevers E (2012) Severe measles, vitamin A deficiency, and the Roma community in Europe. Emerg Infect Dis 18: 1537-1539.

28. Lievano F, Galea SA, Thornton M, Wiedmann RT, Manoff SB, et al. (2012) Measles, mumps, and rubella virus vaccine (M-M-R $\left.{ }^{\mathrm{TM}} \mathrm{II}\right)$ : a review of 32 years of clinical and postmarketing experience. Vaccine 30: 6918-6926. 